\title{
57. Cerebral Serio-Stereo-Angiography, an Apparatus and some Clinical Experiences with it
}

\author{
Toshio Matsubara \\ Department of Neurosurgery and Psychiatry, \\ Okehazama Hospital, Aichi
}

Serial angiographic apparatus and twin roentgen tubes which are placed side by side at a specific distance for stereoscopic viewer, were connected to have "serio-STEREO-angiography" by means of usual single procedure of contrast injection. Twin roentgen tubes were so synchronized with the serial angiographic apparatus, that every other film in the series is exposed by one of the twin tubes and the alternate films by the other of the twin tubes. In this way, film 1 and 2 are stereoscopic pair, film 3 and 4,5 and 6 , and so on are similarly stereoscopic. Strictly speaking, the pair film were exposed with some time interval (about $0.4 \mathrm{sec}$ in this apparatus), i. e., not simultaneously, however, they were practically enough for stereoscopic view.

Stereoscopic observation of cerebral angiograms in cases of intracranial space taking lesion might be beneficial in neuroradiological studies, however, it would be extraordinary important in case in which abnormal vascularities such as neoplastic, regenerative vessels, collateral circuits and vascular malformations are visualized, as such abnormal vascularities are often difficult to study its anatomy even if biplane roentgenograms are taken. In near future, a pair of twin roentgen tubes will be synchronized with the biplane simultaneous serial angiographic apparaius to have multiple studies without multiplying the number of contrast injection.

\section{Brain Tumor and its Serial Cerebral Angiographic Findings}

\author{
Goro Uemura \\ Department of Neurosurgery, Institute of Brain Research, Niigata University
}

There has been many disputes about relations between kinds of brain tumor and cerebral angiographic findings. Twenty-three cases of serial cerebral angiography on patients with brain tumor in our clinic were analysed. They include nine cases of meningioma, seven cases of glioblastoma 
nultiforme, five cases of astrocytoma and two cases of oligodendrogliomà.

1) Meningioma: Most cases show findings as have ever been accepted unt occasionally it is difficult to diagnose with only one angiogram. It is a haracteristic feature that the tumor contour appears after the middle stage If the arterial phase, becomes sharpest in the capillary phase and remains ill the later stage of the venous phase. Of nine cases five show these typical indings, three of which are angiomatous or angioblastic in type, other two tre menigothelial, and fibroblastic respectively.

There are three cases, in which like an astrocytoma tumor contour is ot visualized and tumor vessels are scanty, and they are fibroblastic in type. $\mathrm{n}$ one case both contour and vessels of the tumor ane not visualized at all Ind this is identified histologically as Cushing's type V.

2) Glioblastoma multiforme: In typical three cases tumor contour appears hrough the middle stage of the arterial phase into the capillary phase and lisappears in the latter stage of the venous phase. Other characteristic features Ire ectopic vascular new-growth of various size, microaneurysm, arterio-venous istula, change of the caliber of the vessels.

3) Astrocytoma: In all five cases the tumor is scanty of vascularization ind only meshy fine vessels are visualized around the tumor.

4) Oligodendroglioma: This shows diffeuse tumor contour but might e differentiated from meningioma with some features.

\title{
59. Encephaloscope-Clinical Application (II)
}

\author{
Diagnosis and Treatment of Apoplexy \\ Masahiro Ogata, Kōtaro Tsuda, Tatsuhisa Ono, Reiji HoRide, \\ Toshiaki IsHIKAWA andMikio WATANABE \\ Neurosurgical Section of the Departmeni of Surgery, \\ Kobe Municipal Central Hospital \\ Hiroshi Matsumura \\ 1st Surgical Division, Medical Department, Kyoto University
}

We have completed a new endoscope in April, 1963, which can safely e used in making diagnosis and treatment of intracerebral changes under irect vision. This new instrument is named the "ENCEPHALOSCOPE". he outside diameter of the optical telescope is only $3.1 \mathrm{~mm}$ which is the nest among direct viewing endoscopes in clinical use. It can be safely roceeded into the brain while observing the object closely which is in direct 